\title{
ALGORITMO DE ALOCAÇÃO DE RECURSOS DISCRETOS COM ANÁLISE DE ENVOLTÓRIA DE DADOS
}

\author{
João Carlos Correia Baptista Soares de Mello \\ Departamento de Engenharia de Produção \\ Universidade Federal Fluminense (UFF) \\ Niterói - RJ \\ jcsmello@producao.uff.br
}

Eliane Gonçalves Gomes *

Empresa Brasileira de Pesquisa Agropecuária (Embrapa)

Brasília - DF

eliane.gomes@embrapa.br

Fabiana Rodrigues Leta

Departamento de Engenharia Mecânica

Universidade Federal Fluminense (UFF)

Niterói - RJ

fabiana@ic.uff.br

Maria Helena Campos Soares de Mello

Departamento de Engenharia de Produção

Universidade Federal Fluminense (UFF)

Niterói - RJ

gmamhel@vm.uff.br

* Corresponding author / autor para quem as correspondências devem ser encaminhadas

Recebido em 09/2004; aceito em 11/2005 após 1 revisão

Received September 2004; accepted November 2005 after one revision

\section{Resumo}

A alocação de recursos é um dos problemas clássicos abordados pela Pesquisa Operacional. A inserção de modelos de Análise de Envoltória de Dados (DEA) nesse contexto traz novas possibilidades de estudo, em especial quando combinada com o uso de algoritmos de programação inteira. Este artigo apresenta um algoritmo para distribuição de recursos discretos usando modelos DEA de forma seqüencial. O método proposto é aplicado no estudo de caso da alocação de vagas docentes na Universidade Federal Fluminense. Os resultados são comparados com aqueles obtidos pela comissão encarregada da distribuição das vagas.

Palavras-chave: DEA; alocação de recursos; algoritmo seqüencial.

\begin{abstract}
The resource allocation is one of the main problems in Operational Research. The use of Data Envelopment Analysis (DEA) in this field is a new feature with a great potential, mainly when combined with integer programming problems. This paper presents an algorithm to allocate integer resources using a step-by-step DEA algorithm. We applied the proposed approach to a real case study, which consists in allocating teacher positions in some departments of Universidade Federal Fluminense. We compare the results with those obtained by the official commission.
\end{abstract}

Keywords: DEA; resources allocation; step-by-step algorithm. 


\section{Introdução}

Os modelos de Análise de Envoltória de Dados (Data Envelopment Analysis - DEA) medem a eficiência de unidades produtivas. $\mathrm{O}$ resultado dessa medida serve normalmente para avaliar as unidades e indicar quais as melhores práticas gerenciais em que elas devem se espelhar.

A medida de eficiência pode ser considerada como um índice de aproveitamento de recursos, e, sendo assim, pode ser usada para a alocação e re-alocação de recursos. Devem-se alocar mais recursos para as unidades que melhor fazem uso deles. A quantificação dos recursos para cada unidade pode ser feita de forma proporcional ao índice de eficiência, desde que os recursos possam ser expressos como uma variável contínua. No caso de a variável ser discreta, a simples proporcionalidade não é aplicável, já que se pode atribuir quantidades fracionárias às unidades em avaliação.

Este artigo apresenta um modelo seqüencial de atribuição de recursos em modelos DEA, inspirado no modelo de votação de Hondt (Shuster et al., 2003), considerando-se que o excesso de recursos a ser distribuído tem soma constante. Caso fosse de interesse realocar os recursos já existentes, mantendo-se constante o total dos recursos (soma dos recursos constante) poderia ser usado o modelo DEA com Ganhos de Soma Zero - DEA-GSZ (para os casos CCR e BCC) (Gomes et al., 2004, 2003; Gomes, 2003; Estellita Lins et al., 2003), o modelo DEA hiperbólico (para o caso CCR) (Avellar, 2004) ou o modelo de alocação centralizada de recursos (para o caso BCC) (Lozano \& Villa, 2004). No entanto, estes modelos são válidos apenas para o caso de recursos com distribuição contínua, enquanto o método aqui proposto tem validade para recursos medidos por números inteiros.

$\mathrm{O}$ algoritmo seqüencial de alocação de recursos em DEA proposto neste artigo é aplicado à distribuição de vagas docentes aos departamentos de ensino do Centro Tecnológico da Universidade Federal Fluminense (UFF). O modelo considera o número de professores de cada departamento, o envolvimento com atividades de ensino e pesquisa e a existência de projetos de expansão aprovados. São usados dados da Comissão Provisória de Alocação de Vagas Docentes (CPAVD) da UFF.

\section{Análise de Envoltória de Dados}

A Análise de Envoltória de Dados (Data Envelopment Analysis - DEA) tem como objetivo medir a eficiência de unidades tomadoras de decisão, designadas por DMUs (Decision Making Units), na presença de múltiplos inputs (entradas, recursos ou fatores de produção) e múltiplos outputs (saídas ou produtos).

Há dois modelos DEA clássicos: CCR e BCC. O modelo CCR (também conhecido por CRS ou constant returns to scale), trabalha com retornos constantes de escala (Charnes et al., 1978). Em sua formulação matemática, considera-se que cada DMU $k, k=1, \ldots, n$, é uma unidade de produção que utiliza $m$ inputs $x_{i k}, i=1, \ldots, m$, para produzir $s$ outputs $y_{j k}$, $j=1, \ldots, s$. Esse modelo maximiza o quociente entre a combinação linear dos outputs e a combinação linear dos inputs, com a restrição de que para qualquer DMU esse quociente não pode ser maior que 1.

Mediante alguns artifícios matemáticos, esse modelo pode ser linearizado, transformando-se em um Problema de Programação Linear (PPL) apresentado em (I), onde $h_{o}$ é a eficiência da DMU $o$ em análise; $x_{i o}$ e $y_{j o}$ são os inputs e outputs da DMU $o ; v_{i}$ e $u_{j}$ são os pesos calculados pelo modelo para inputs e outputs, respectivamente. 


$$
\begin{aligned}
& \max h_{o}=\sum_{j=1}^{s} u_{j} y_{j o} \\
& \text { sujeito a } \\
& \sum_{i=1}^{m} v_{i} x_{i o}=1 \\
& \sum_{j=1}^{s} u_{j} y_{j k}-\sum_{i=1}^{m} v_{i} x_{i k} \leq 0, \quad \forall k \\
& u_{j}, v_{i} \geq 0 \quad \forall i, j
\end{aligned}
$$

O modelo BCC (Banker et al., 1984), também chamado de VRS (variable returns to scale), considera situações de eficiência de produção com variação de escala e não assume proporcionalidade entre inputs e outputs.

Embora os modelos DEA tenham a vantagem de permitir fazer ordenações sem depender de opiniões de decisores, são extremamente benevolentes com as unidades avaliadas. Estas podem ser eficientes desconsiderando várias das variáveis de avaliação. Assim, é comum haver um grande número de DMUs com eficiência 100\%. Entretanto, quando há preferências entre os inputs e/ou outputs por parte dos agentes de decisão, esses julgamentos são incorporados aos modelos DEA através de restrições aos pesos (ou multiplicadores) associados aos inputs e/ou aos outputs das unidades avaliadas. Allen et al. (1997) apresentam uma completa revisão da evolução da incorporação de julgamentos de valor através de restrições aos pesos, enquanto Angulo Meza \& Estellita Lins (2002) apresentam uma revisão desse e de outros métodos de melhorar ordenações em DEA.

A incorporação de julgamentos de valor através de restrições aos pesos pode ser dividida em três grupos de métodos (Estellita Lins \& Angulo Meza, 2000): restrições diretas sobre os multiplicadores; ajuste dos níveis de input-output observados para a captura de julgamentos de valor; restrição a inputs e outputs virtuais.

\section{Alocação de Recursos em DEA}

A alocação de recursos é um dos problemas clássicos abordados pela Pesquisa Operacional, em especial, pelos modelos de programação matemática. A inserção de DEA nesse contexto traz a possibilidade de considerar os planos de produção viáveis e de verificar os tradeoffs entre inputs e outputs, com base na caracterização do conjunto de possibilidades de produção (Korhonen \& Syrjänen, 2001).

O objetivo de um controle central ao alocar recursos às unidades constituintes é que a produção global seja maximizada, ou seja, a questão da alocação dos recursos (inputs) está intrinsecamente ligada na prática ao estabelecimento de alvos para a produção (outputs) (Beasley, 2001).

Na literatura, sob o tema "alocação de recursos em DEA" são encontrados, basicamente, dois segmentos de pesquisa: alocação de recursos e estabelecimento de alvos. A determinação de alvos é a especificação de níveis de inputs ou outputs para cada DMU isoladamente, independentemente do contexto organizacional em que esteja inserida (sem considerar a limitação dos recursos). 
A alocação de recursos refere-se à determinação dos níveis de inputs ou outputs, quando a organização tem limitados recursos de inputs ou possibilidades de outputs. Neste caso, os níveis das variáveis devem ser estabelecidos simultaneamente para que as limitações de recursos sejam respeitadas.

\subsection{Estado da arte}

Um procedimento em cinco etapas para a alocação de inputs é apresentado por Golany et al. (1993). Os recursos são distribuídos de acordo com as eficiências (ponderadas) das DMUs. Este estudo, também classificado como de determinação de alvos, usa o modelo DEA aditivo (Charnes et al., 1985).

Para o caso de output único, Golany \& Tamir (1995) apresentam um modelo de alocação de recursos que determina simultaneamente os alvos para inputs e outputs, com base na maximização total do output.

Athanassopoulos $(1995,1998)$ apresenta dois modelos de alocação de recursos. O primeiro é baseado em programação por metas e DEA, e a fronteira de eficiência é caracterizada de maneira semelhante ao modelo DEA do envelope. As DMUs neste modelo são apresentadas de maneira global com relação aos alvos organizacionais. No segundo modelo, o conjunto de possibilidades de produção é apresentado com restrições, o que lembra o modelo DEA dos multiplicadores.

Para o caso de input único, Thanassoulis (1996) propõe um modelo de programação inteira mista, que agrupa as unidades em conjuntos e determina um nível marginal de recursos. Este fator é usado pelos decisores para alocar o recurso. Em outro artigo (Thanassoulis, 1998), o mesmo autor estima (por regressão e programação linear) um conjunto único de fatores que são aplicadas a todas as DMUs, ainda considerando-se o caso de input único.

Beasley (2001) apresenta um modelo de alocação de recursos que partem da reinterpretação de DEA como sendo um modelo não linear único visto do nível organizacional. Neste modelo, os pesos são escolhidos simultaneamente para todas as DMUs, maximizando a eficiência média.

O problema da alocação de recursos em um ambiente de decisão centralizado, no qual o interesse é maximizar os outputs que cada unidade pode produzir com os recursos disponíveis, é tratado por Korhonen \& Syrjänen (2001). Os autores utilizam DEA e programação linear multiobjetivo na escolha do plano de alocação mais preferido. Lozano \& Villa (2004) discutem igualmente a tomada de decisão centralizada em relação à alocação de recursos e propõem um modelo DEA BCC no qual a maximização de eficiência de cada DMU individual é feita simultaneamente à minimização do total de recursos ou maximização da produção total.

Yan et al. (2002) discutem um modelo DEA inverso (Wei et al., 2000) com restrições de preferência sobre as variáveis, no qual a DMU estima os níveis de inputs/outputs quando da revisão dos níveis passados, dada a eficiência atual. $\mathrm{O}$ modelo proposto é aplicado à realocação dos recursos em um grupo de produtores de aparelhos elétricos.

O caso de alocação de recursos em que seja necessário admitir que o total não pode ser alterado é estudado por alguns autores. Em Gomes et al. (2004, 2003), Gomes (2003) e Estellita Lins et al. (2003) é proposto o modelo DEA com Ganhos de Soma Zero (DEA-GSZ), 
no qual a restrição dos modelos DEA clássicos de total liberdade de produção ou de uso dos recursos é substituída pela restrição de soma total constante de inputs ou outputs. Assim, nos modelos DEA-GSZ, CCR ou BCC, uma DMU ineficiente que busque a fronteira pelo aumento de outputs (redução de inputs) imputará às demais a redução do valor de seus outputs (ou aumento de seus inputs) de modo a manter a soma total constante. Assim, os modelos DEA-GSZ têm aplicação direta nos estudos de alocação ou (re)alocação de recursos (ou de produção), em que a restrição de soma constante seja uma necessidade da modelagem.

Avellar (2004) propõe quatro modelos tipo DEA baseados em inputs/outputs limitados, nos quais a distribuição dos recursos/produtos pode ser influenciada tanto pelos inputs como pelos outputs envolvidos. A construção dos modelos tomou como base o perfil geométrico da fronteira CCR tridimensional, que é substituída por uma fronteira hiperbólica ou esférica, função da natureza da variável a distribuir.

\section{Algoritmo Seqüencial para Alocação de Recursos em DEA}

Os modelos citados no item anterior fazem realocação de recursos já existentes. Em sua maioria, não consideram a possibilidade de os recursos serem discretos. A proposta deste artigo é desenvolver um algoritmo que distribua novos recursos discretos entre as unidades, com base nas suas eficiências.

Uma primeira abordagem que se poderia pensar para resolver o problema acima seria a distribuição dos recursos disponíveis apenas para as unidades eficientes. Por ser extremamente simplista e apresentar alguns problemas, não foi encontrada nenhuma menção a esta técnica na literatura. O primeiro problema desta abordagem é que a quantidade de recursos a serem distribuídos pode não ser múltiplo do número de DMUs eficientes, o que obrigaria a algum tipo de ordenação entre as unidades de eficiência 100\%. Outro problema é que esta alocação desconsidera a existência de DMUs ineficientes mas muito próximas da eficiência. Pode não ser conveniente atribuir todos os recursos às unidades eficientes e não atribuir nada a uma DMU com, por exemplo, $99 \%$ de eficiência.

Este é um problema semelhante ao da transformação de votos em vagas para deputados. Um dos métodos de resolver este problema é o algoritmo de Hondt (Shuster et al., 2003), que preenche as vagas de forma seqüencial. $\mathrm{O}$ algoritmo atribui uma vaga de cada vez, segundo determinados indicadores. No entanto, o algoritmo de Hondt, por ser aplicado ao total de votos, parte do princípio que a soma dos percentuais de votação é $100 \%$. Ora, a soma das eficiências de um conjunto de DMUs é, percentualmente, sempre superior a $100 \%$, invalidando a aplicação do método de Hondt. Outras formas de resolver este problema são os métodos baseados em sobras, que também exigem que a soma dos percentuais seja $100 \%$. Uma revisão geral destes métodos e de suas propriedades é encontrada em Maia (2005).

O algoritmo proposto neste artigo segue uma filosofia semelhante, cujas etapas são apresentadas na Figura 1.

No algoritmo proposto, os recursos são distribuídos a cada conjunto de DMUs eficientes, mas apenas uma unidade de recurso para cada DMU eficiente. Feita esta distribuição, supõese que os recursos já foram efetivamente distribuídos e calculam-se as novas DMUs eficientes, repetindo-se o algoritmo até distribuir todos os recursos. O objetivo deste passo é verificar qual seria o comportamento das DMUs, se estas mantivessem a mesma produção, mas pudessem contar com os recursos agora distribuídos. Desta forma, o algoritmo proposto 
é um algoritmo seqüencial de distribuição de recursos, que toma como base para a alocação as eficiências calculadas pelos modelos DEA.

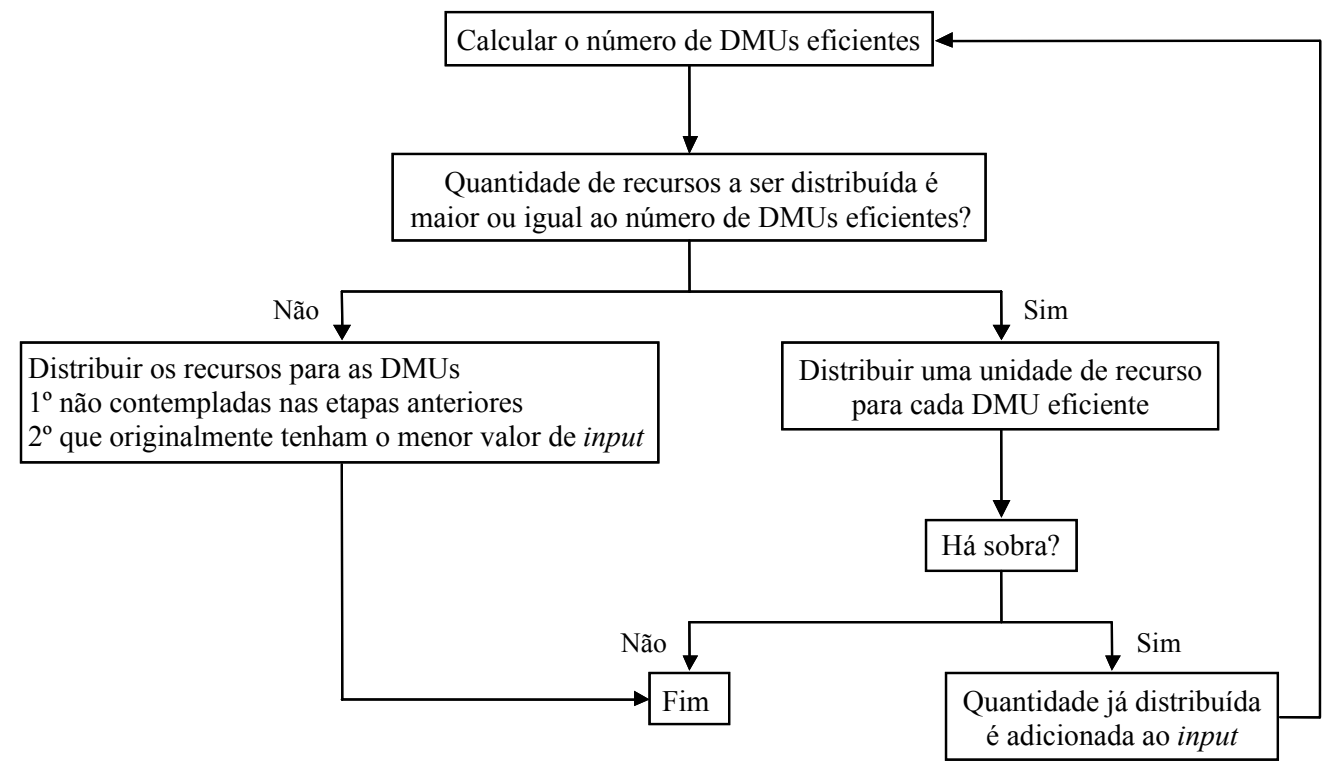

Figura 1 - Etapas do algoritmo seqüencial de alocação de recursos em modelos DEA.

Especial atenção deve ser dada ao caso em que a quantidade de recursos a ser distribuída for inferior ao número de DMUs eficientes, em determinada etapa do processo. Neste caso, propõe-se que os recursos sejam distribuídos, em primeiro lugar, para as unidades eficientes que não foram contempladas nas etapas anteriores e, em seguida, para as unidades eficientes com menor quantidade original do recurso (input) em questão. A primeira das regras justifica-se pela decisão de fazer uma distribuição mais igualitária. Já a segunda, usa o principio que a mesma unidade de recurso é mais útil para uma unidade que tenha menos recursos do que para uma que tenha mais. Ou seja, admite que a segunda derivada da função utilidade é negativa, o que é um pressuposto geralmente aceito em situações de ganhos (Valadares Tavares et al., 1996).

$\mathrm{O}$ uso da quantidade original como fator de desempate parte do princípio de que os recursos são todos distribuídos no mesmo instante, isto é, as etapas intermediárias do algoritmo são apenas ferramentas para obter-se o resultado final. Caso os recursos sejam distribuídos em momentos distintos, com espaçamento temporal apreciável, a informação da quantidade original de recursos perde sentido. Em um caso como este, poder-se-ia usar como critério de desempate, a quantidade de recursos que a DMU dispõe naquela iteração.

$\mathrm{O}$ algoritmo proposto resolve totalmente um dos problemas da abordagem simplista e resolve o outro na grande maioria dos casos. De fato, se após a primeira iteração (quando são distribuídos recursos apenas para as unidades originalmente eficientes) houver sobra de recursos, estes são distribuídos de acordo com a eficiência que considere a distribuição anterior. Com isto, DMUs de eficiência próxima a $100 \%$ podem tornar-se agora $100 \%$ eficientes e serem elas a receber recursos. O segundo problema (haver mais DMUs eficientes 
do que recursos a distribuir) é resolvido, na maioria dos casos, com o critério de desempate apresentado. Entretanto, este critério não é exaustivo, ou seja, após sua aplicação podem subsistir empates. Nesta situação, há necessidade de um critério de desempate adicional, que deverá ser estabelecido, caso a caso, segundo as estruturas de preferências dos decisores.

\section{Estudo de Caso}

\subsection{Problema}

Em 2001 o Conselho Universitário da Universidade Federal Fluminense determinou que a Comissão Provisória de Alocação de Vagas Docentes (CPAVD) estabelecesse critérios acadêmicos para a alocação de vagas docentes da carreira do magistério superior nos departamentos de ensino. Esta comissão deveria então elaborar uma proposta de distribuição de vagas, baseada nos seguintes critérios preestabelecidos (UFF, 2001a):

- No mínimo $60 \%$ considerando a relação professor/aluno e a carga horária didática;

- Até 30\% segundo a produção acadêmica;

- Até $20 \%$ segundo os projetos de expansão aprovados.

A proposta detalha, ainda, que:

- A relação professor/aluno e a carga horária didática só deverão levar em conta atividades presenciais e gratuitas, nas quais os professores não recebam pró-labore;

- Na produção acadêmica departamental deverão ser considerados os dados constantes no Relatório SAD/RADOC relativos a 1999 e 2000;

- Na análise dos projetos de expansão sejam considerados os pleitos acadêmicos relativos à criação de cursos, habilitações, áreas de concentração, campos de confluência, ampliação de turmas ou turnos, reformulações curriculares, entre outros, desde que aprovados pelos Conselhos Superiores da universidade.

A relação professor/aluno é calculada considerando-se dois indicadores: professores do quadro permanente da carreira do magistério superior, lotados e em exercício no respectivo departamento, e os estudantes regularmente matriculados e inscritos em disciplinas ministradas presencialmente por professores do respectivo departamento.

A carga horária didática departamental é expressa pela carga horária total das disciplinas ministradas pelo departamento, presenciais e gratuitos.

Para o cálculo da produção acadêmica, a CPAVD utilizou a base de dados da GED (Gratificação de Estímulo à Docência), com as seguintes considerações (UFF, 2001b):

- Utilizar os pesos já aprovados no Conselho de Ensino e Pesquisa que fazem parte da pontuação de produção para a GED;

- Retirar os itens: artigos de opinião, publicações em veículos de circulação local, trabalhos apresentados em Congressos Científicos, Resumos publicados em Congressos Científicos, textos didáticos para uso local e participações em Exposições ou Apresentações artísticas;

- Manter os itens: capítulos de livros publicados, artigos de divulgação científica, filmes (vídeos) artísticos, filmes (vídeos) científicos, livros publicados, artigos em 
periódicos internacionais, artigos em periódicos internacionais indexados, artigos em periódicos nacionais, artigos em periódicos nacionais indexados, processos desenvolvidos com ou sem patente, produtos desenvolvidos com ou sem patente, teses doutorado defendidas e aprovadas, teses doutorado orientadas e aprovadas, teses mestrado defendidas e aprovadas, teses mestrado orientadas e aprovadas e trabalhos publicados em anais de congressos;

- Utilizar apenas os dados relativos ao ano de 2000, já que os dados relativos ao ano de 1999 revelaram-se incompletos para a Comissão, não diferenciando os periódicos indexados dos não-indexados, o que provoca uma discrepância de difícil correção.

Uma vez calculado o total da produção acadêmica de cada departamento, foi feita a divisão pelo número de docentes efetivos de cada departamento. Em seguida foi feita uma ordenação decrescente até o número de vagas a ser distribuído por este critério. Essa hierarquia serviu de base para a distribuição das vagas docentes.

Essa abordagem apresenta alguns inconvenientes. O primeiro é que na prática esta é uma abordagem de pesos fixos, não possibilitando a avaliação de cada departamento segundo suas maiores necessidades. O segundo é que é uma avaliação estática, já que distribui todos os recursos considerando a situação em um determinado instante. Não leva, portanto, em conta o fato de que a distribuição do primeiro recurso já alterou toda a situação que havia sido avaliada. O modelo DEA corrige o primeiro inconveniente; o uso do algoritmo seqüencial proposto neste artigo, o segundo.

\subsection{Modelagem}

Como exposto na descrição do problema, as DMUs são os departamentos de ensino do Centro Tecnológico da UFF, no total de 12 unidades de avaliação.

Uma vez que os recursos a serem distribuídos são vagas para abertura de concurso para docentes, o input único é o número de docentes alocados em cada departamento. Os outputs devem estar relacionados ao trabalho desses professores e são representados pelo número de alunos.hora equivalente atendidos pelo departamento, produção científica (conforme ponderação aprovada pelo Conselho de Ensino e Pesquisa da UFF) e a existência de projetos de expansão aprovados pelos colegiados superiores da universidade.

O output "projetos de expansão" é uma variável binária, recebendo valor 1 caso haja projeto aprovado, e zero caso contrário. "Aluno.hora equivalente" refere-se ao número de alunos multiplicado pelo número de horas.aula de cada um, dividido por um coeficiente estipulado pelo Ministério da Educação (MEC), que se destina a equiparar as várias áreas do conhecimento. No caso em estudo, esse coeficiente foi igual para todos os departamentos.

Além destes, que foram considerados pela CPAVD, considerou-se ainda o output número de disciplinas de cada departamento. A inclusão desta variável tem como objetivo assegurar que departamentos que ofereçam muitas disciplinas nas quais se inscrevem poucos alunos possam ter condições mínimas de atendimento aos discentes.

A Tabela 1 apresenta os dados utilizados neste artigo, na qual TAU $=$ Departamento de Arquitetura; TCC $=$ Departamento de Ciência da Computação; TDT $=$ Departamento de Desenho Técnico; TEC $=$ Departamento de Engenharia Civil; TEE $=$ Departamento de Engenharia Elétrica; TEM = Departamento de Engenharia Mecânica; TEP $=$ Departamento 
de Engenharia de Produção; TEQ = Departamento de Engenharia Química; TET = Departamento de Engenharia de Telecomunicações; TMC = Departamento de Ciência dos Materiais; TMI = Departamento de Metalurgia Industrial; TUR $=$ Departamento de Urbanismo.

Tabela 1 - DMUs, input e outputs para o estudo de caso.

\begin{tabular}{cccccc}
\hline Departamento & Docentes & Alunos.hora & $\begin{array}{c}\text { Projetos de } \\
\text { expansão }\end{array}$ & Disciplinas & $\begin{array}{c}\text { Produção } \\
\text { científica }\end{array}$ \\
\hline TAU & 25 & 155,9 & 0 & 29 & 13,1 \\
TCC & 43 & 456,7 & 0 & 51 & 26,3 \\
TDT & 11 & 86,7 & 0 & 12 & 15,2 \\
TEC & 61 & 406,4 & 1 & 99 & 14,1 \\
TEE & 30 & 79,4 & 0 & 43 & 12,8 \\
TEM & 24 & 157 & 0 & 40 & 44,8 \\
TEP & 33 & 415,2 & 0 & 38 & 42,4 \\
TEQ & 22 & 108,0 & 0 & 44 & 5,5 \\
TET & 33 & 317,0 & 0 & 50 & 6,6 \\
TMC & 18 & 122,6 & 1 & 29 & 5,4 \\
TMI & 22 & 116,7 & 1 & 33 & 8,8 \\
TUR & 14 & 115,8 & 0 & 23 & 18,1 \\
\hline
\end{tabular}

Foi usado o modelo DEA CCR, com restrições aos pesos. A escolha do modelo CCR ao invés de BCC justifica-se por este atribuir eficiência 100\% para unidades com menor input e maior output, independente da relação entre eles. Neste caso, um departamento poderia receber vagas de professor só porque é de porte muito grande ou muito pequeno.

A modelagem proposta mede o quanto um departamento está sobrecarregado: quanto mais eficiente é uma DMU, mais trabalho têm os seus professores.

As restrições destinam-se a adequar o modelo DEA clássico, que dá total liberdade aos pesos, às determinações do Conselho Universitário da UFF (UFF, 2001a), que obrigam a que o maior número de vagas seja distribuído considerando-se as atividades didáticas, em seguida a produção científica e, por último, os projetos de expansão aprovados. Neste artigo, diferentemente do usado em Soares de Mello et al. (2004) e Leta et al. (2003), são usadas restrições aos pesos do tipo Cone Ratio (Charnes et al., 1990). Esse tipo de restrição dá maior liberdade de otimização a cada DMU, ao contrário das restrições do tipo input ou output virtual que são, muitas vezes, quase um esquema de pesos fixos e que conduzem, freqüentemente, a inviabilidades dos PPLs (Soares de Mello et al., 2002). As inviabilidades provocadas pelas restrições do tipo Cone Ratio são mais raras. As condições em que estas ocorrem são estudadas em Estellita Lins \& Silva (2001).

As restrições aos pesos dos multiplicadores usadas neste artigo indicam que, após a normalização das variáveis, o peso atribuído à quantidade de alunos.hora é maior que o peso atribuído à variável pesquisa realizada. Já o peso dado ao número de disciplinas é também maior que o peso dado à pesquisa realizada, e este peso é maior que o peso atribuído aos projetos de expansão. As inequações apresentadas em (II) formalizam as restrições descritas. 


$$
\begin{aligned}
& u_{\text {alunos.hora }} \geq u_{\text {pesquisa }} \\
& u_{\text {disciplinas }} \geq u_{\text {pesquisa }} \\
& u_{\text {pesquisa }} \geq u_{\text {projetos de expansão }}
\end{aligned}
$$

Para obter resultados foi utilizado o software SIAD (Angulo Meza et al., 2004), que já tem a opção de normalização prévia das variáveis de forma que os multiplicadores reflitam realmente a importância da variável a eles associada.

\subsection{Resultados}

A aplicação do algoritmo detectou os departamentos TEM, TEP, TEQ, TET, TMC e TUR como departamentos inicialmente eficientes, conforme a Tabela 2. Verifica-se que o número de DMUs eficientes é inferior ao número de vagas a distribuir. Estas DMUs recebem, de acordo com o algoritmo proposto, as primeiras vagas docentes (uma unidade de input), ou seja, 6 vagas do total de 15 a serem distribuídas aos departamentos do Centro Tecnológico (CTC).

Tabela 2 - Resultados da $1^{\text {a }}$ rodada do modelo DEA CCR com restrições aos pesos do tipo Cone Ratio.

\begin{tabular}{cc}
\hline DMU & Eficiência (\%) \\
\hline TAU & 72,1 \\
TCC & 92,4 \\
TDT & 83,9 \\
TEC & 92,7 \\
TEE & 73,1 \\
TEM & 100,0 \\
TEP & 100,0 \\
TEQ & 100,0 \\
TET & 100,0 \\
TMC & 100,0 \\
TMI & 91,4 \\
TUR & 100,0 \\
\hline
\end{tabular}

Ainda de acordo com o algoritmo da Figura 1, a próxima etapa é calcular o modelo DEA já com o novo valor do número de vagas. As DMUs eficientes (as mesmas da $1^{\text {a }}$ iteração) recebem uma unidade do input "docentes" e o modelo DEA é rodado novamente. Até esta etapa haviam sido distribuídas 12 vagas, 2 para cada uma das seguintes DMUS: TEM, TEP, TEQ, TET, TMC e TUR. Na $3^{\text {a }}$ rodada, o número de DMUs eficientes é maior que o número de vagas restantes. Assim, conforme o algoritmo recebem vagas as DMUs eficientes que não receberam vagas nas etapas anteriores (TEC e TMI) e, depois, aquela com menor valor inicial do input (TMC).

Os resultados da aplicação dessas fases do algoritmo seqüencial de alocação de recursos em DEA encontram-se resumidos na Tabela 3. A Tabela 4 compara a distribuição de vagas docentes proposta por dois métodos: o algoritmo proposto neste artigo e a distribuição feita pela CPAVD. 
Tabela 3 - Resultados das fases seguintes do algoritmo seqüencial de alocação de recursos em DEA.

\begin{tabular}{ccc}
\hline \multirow{2}{*}{ DMU } & \multicolumn{2}{c}{ Eficiência (\%) por rodada } \\
\cline { 2 - 3 } & $\mathbf{2}^{\mathbf{a}}$ & $\mathbf{3}^{\mathbf{a}}$ \\
\hline TAU & 75,5 & 78,2 \\
TCC & 95,2 & 98,0 \\
TDT & 88,9 & 90,0 \\
TEC & 98,4 & 100,0 \\
TEE & 76,4 & 80,0 \\
TEM & 100,0 & 100,0 \\
TEP & 100,0 & 100,0 \\
TEQ & 100,0 & 100,0 \\
TET & 100,0 & 100,0 \\
TMC & 100,0 & 100,0 \\
TMI & 96,4 & 100,0 \\
TUR & 100,0 & 97,3 \\
\hline
\end{tabular}

Tabela 4 - Distribuição das vagas docentes segundo duas distintas abordagens.

\begin{tabular}{ccc}
\hline DMU & Algoritmo seqüencial & CPAVD \\
\hline TAU & 0 & 0 \\
TCC & 0 & 2 \\
TDT & 0 & 1 \\
TEC & 1 & 1 \\
TEE & 0 & 0 \\
TEM & 2 & 3 \\
TEP & 2 & 3 \\
TEQ & 2 & 0 \\
TET & 2 & 1 \\
TMC & 3 & 1 \\
TMI & 1 & 1 \\
TUR & 2 & 2 \\
\hline
\end{tabular}

Na Tabela 4, as 15 vagas foram distribuídas pela CPAVD da seguinte forma, de modo a respeitar a importância relativa entre os critérios de avaliação segundo definição do Conselho de Ensino e Pesquisa: 10 vagas pelo critério aluno.hora, 3 vagas pelo critério produção científica e 2 pelo critério projetos de expansão. Estas últimas foram atribuídas a TEC, TMC e TMI pela implantação de novos cursos na unidade da UFF em Volta Redonda. As vagas do critério produção científica foram alocadas aos três departamentos melhor colocados neste item, a saber, TEM e TEP. A alocação de vagas pelo critério aluno.hora foi feita em duas etapas. Na primeira foi verificado quais departamentos atendiam ao mínimo de onze horas semanais de carga horária por docente e tinham saldo positivo entre a necessidade docente, conforme estabelecido pelo MEC (Ministério da Educação, 2001), e o número efetivo de 
docentes. Atenderam a esta exigência 6 departamentos (TCC, TDT, TEM, TEP, TET e TUR), tendo recebido cada um deles, inicialmente, 1 vaga docente. As quatro vagas restantes foram atribuídas aos departamentos com maior carga horária média por professor, TCC, TEM, TEP e TUR.

A análise da Tabela 4 mostra o método proposto levou em conta fortes especificidades de alguns departamentos, ignoradas pela CPAVD. Ao dar mais liberdade de atribuição de pesos (sem ficarem inteiramente livres), o método aqui usado permitiu a atribuição de vagas docentes ao departamento de Engenharia Química, que tem grande número de disciplinas. Também, aumentou as vagas do departamento de Ciência dos Materiais, que suporta grande parte da carga horária decorrente da implantação dos cursos de Engenharia Mecânica e Engenharia de Produção em Volta Redonda.

Deve-se observar que o algoritmo proposto atribuiu recursos a duas DMUs, TEC e TMI, que originalmente não eram eficientes, o que comprova que esta abordagem resolver o problema apontado no início do item 4.

\section{Conclusões}

Este artigo apresentou uma abordagem alternativa para a alocação de recursos, que faz uso de modelos DEA aplicados e atualizados seqüencialmente.

Os resultados sugerem que é essencial haver critérios embasados tecnicamente para a distribuição de recursos no setor público. O método proposto evitou pontos de corte arbitrários. O modelo usado pela CPAVD assemelha-se a modelos eleitorais baseados em quocientes de corte, enquanto o algoritmo proposto neste artigo permite a distribuição de vagas sem pré-exclusão alguma. Quando aplicados a resultados eleitorais, Maia (2005) mostrou que os métodos sem exclusão são mais fidedignos.

O método deste artigo respeitou a relação entre importância de critérios, mas incluiu um parâmetro extra (número de disciplinas obrigatórias) para melhor medir a carga didática dos professores. Dessa forma, foi considerada a crítica do departamento de Engenharia Elétrica, que afirma ter poucos alunos, mas muitas turmas. Mesmo com a nova variável com a liberdade de otimização permitida pelo modelo DEA, este departamento não recebeu nenhum recurso extra, comprovando que realmente trabalha com excesso de professores.

Os próximos desenvolvimentos devem contemplar a imposição de restrições sobre limites máximo e mínimo de recursos alocados, bem como a desagregação do índice de produtividade acadêmica. Há ainda a intenção de aplicar o método à distribuição de outros tipos de recursos, bem como aprofundar o estudo sobre os pesos usados e sobre o uso de outros tipos de métodos de restrições aos pesos em DEA.

\section{Agradecimentos}

Ao CNPq pelo apoio financeiro, processo 471014/2003-6, e aos revisores pelas valiosas contribuições. 


\section{Referências Bibliográficas}

(1) Allen, R.; Athanassopoulos, A.; Dyson, R.G. \& Thanassoulis, E. (1997). Weights restrictions and value judgements in data envelopment analysis: evolution, development and future directions. Annals of Operations Research, 73, 13-34.

(2) Angulo Meza, L. \& Estellita Lins, M.P. (2002). Review of Methods for Increasing Discrimination in Data Envelopment Analysis. Annals of Operations Research, 116, 225-242.

(3) Angulo Meza, L.; Biondi Neto, L.; Soares de Mello, J.C.C.B.; Gomes, E.G. \& Coelho, P.H.G. (2004). FSDA - Free Software for Decision Analysis (SLAD Software Livre de Apoio a Decisão): A Software Package for Data Envelopment Analysis Models. $12^{\circ}$ Congreso Latino-Iberoamericano de Investigación Operativa y Sistemas, La Habana, Cuba.

(4) Athanassopoulos, A.D. (1995). Goal programming \& data envelopment analysis (GoDEA) for target-based multi-level planning: Allocating central grants to the Greek local authorities. European Journal of Operational Research, 87, 535-550.

(5) Athanassopoulos, A.D. (1998). Decision Support for Target-Based Resource Allocation of Public Services in Multiunit and Multilevel Systems. Management Science, 44(2), 173-187.

(6) Avellar, J.V.G. (2004). Modelos DEA com soma constante de inputs/outputs. Tese de Mestrado, Instituto Tecnológico de Aeronáutica, São José dos Campos.

(7) Banker, R.D.; Charnes, A. \& Cooper, W.W. (1984). Some models for estimating technical scale inefficiencies in Data Envelopment Analysis. Management Science, 30(9), 1078-1092.

(8) Beasley, J.E. (2001). Allocating fixed costs and resources via data envelopment analysis. Technical Report, The Management School, Imperial College, London. Disponível em $<$ http://mscmga.ms.ic.ac.uk/jeb/jeb.html $>$.

(9) Charnes, A.; Cooper, W.W. \& Rhodes, E. (1978). Measuring the efficiency of decisionmaking units. European Journal of Operational Research, 2, 429-444.

(10) Charnes, A.; Cooper, W.W.; Golany, B.; Seiford, L. \& Stutz, J. (1985). Foundations of data envelopment analysis for Pareto-Koopmans efficient empirical production functions. Journal of Econometrics, 30, 91-107.

(11) Charnes, A.; Cooper, W.W.; Huang, Z.M. \& Sun, D.B. (1990). Polyhedral cone-ratio DEA models with an illustrative application to large commercial banks. Journal of Econometrics, 46, 73-91.

(12) Estellita Lins, M.P. \& Angulo Meza, L. (2000). Análise Envoltória de Dados e perspectivas de integração no ambiente de Apoio à Decisão. Editora COPPE/UFRJ, Rio de Janeiro, 53-64.

(13) Estellita Lins, M.P. \& Silva, A.C.M. (2001). Evitando a inviabilidade em modelos DEA com restrições aos pesos. Relatório Técnico EP03/01-PO, Programa de Engenharia de Produção, COPPE/UFRJ, Rio de Janeiro. 
(14) Estellita Lins, M.P.; Gomes, E.G.; Soares de Mello, J.C.C.B. \& Soares de Mello, A.J.R. (2003). Olympic ranking based on a Zero Sum Gains DEA model. European Journal of Operational Research, 148(2), 312-322.

(15) Golany, B. \& Tamir, E. (1995). Evaluating Efficiency-Effectiveness-Equality Trade-Offs: A Data Envelopment Analysis Approach. Management Science, 41(7), $1172-1184$

(16) Golany, B.; Phillips, F.Y. \& Rousseau, J.J. (1993). Models for improved effectiveness based on DEA efficiency results. IIE Transactions, 25, 2-10.

(17) Gomes, E.G. (2003). Modelos de Análise de Envoltória de Dados com Ganhos de Soma Zero. Tese de Doutorado, Programa de Engenharia de Produção, COPPE/UFRJ, Rio de Janeiro, Dezembro.

(18) Gomes, E.G.; Soares de Mello, J.C.C.B. \& Estellita Lins, M.P. (2003). Busca seqüencial de alvos intermediários em modelos DEA com soma de outputs constante. Investigação Operacional, 23(2), 163-178.

(19) Gomes, E.G.; Soares de Mello, J.C.C.B. \& Estellita Lins, M.P. (2004). Redistribuição de inputs e outputs em modelos de análise de envoltória de dados com ganhos de soma zero. Pesquisa Operacional, 24(2), 269-284.

(20) Korhonen, P. \& Syrjänen, M. (2001). Resource allocation based on efficiency analysis. Working Paper W-293, Helsinki School of Economics and Business Administration, $25 \mathrm{p}$.

(21) Leta, F.R.; Gomes, E.G.; Soares de Mello, M.H.C. \& Soares de Mello, J.C.C.B. (2003). Análise de Envoltória de Dados para Alocação de Recursos: Uma Proposta de Algoritmo Seqüencial. Anais do XXXV Simpósio Brasileiro de Pesquisa Operacional, Natal.

(22) Lozano, S.N. \& Villa, G. (2004). Centralized Resource Allocation Using Data Envelopment Analysis. Journal of Productivity Analysis, 22, 143-161.

(23) Maia, C.C. (2005). Modelos matemáticos em eleições proporcionais. Dissertação de Mestrado, Programa de Engenharia de Produção, UFF, Niterói, Fevereiro.

(24) Ministério da Educação (2001). Portaria 1980. Setembro.

(25) Shuster, K.; Pukelsheim, F.; Drton, M. \& Draper, N.R. (2003). Seat biases of apportionment methods for proportional representation. Electoral Studies, 22(4), 651-676.

(26) Soares de Mello, J.C.C.B.; Estellita Lins, M.P.E.; Soares de Mello, M.H.C. \& Gomes, E.G. (2002). Evaluating the Performance of Calculus Classes Using Operational Research Tools. European Journal Of Engineering Education, 27(2), 209-218.

(27) Soares de Mello, M.H.C.; Leta, F.R.; Gomes, E.G. \& Soares de Mello, J.C.C.B. (2004). Análise envoltória de dados para avaliação de departamento de ensino. Ensaio Avaliação e Políticas Públicas em Educação, 12(42), 611-622.

(28) Thanassoulis, E. (1996). A data envelopment analysis approach to clustering operating units for resource allocation purposes. Omega, 24, 463-476. 
(29) Thanassoulis, E. (1998). Estimating efficient marginal resource levels using data envelopment analysis. Journal of Cost Analysis, 29-52.

(30) UFF (2001a). Alocação de Vagas Docentes. Resolução No. 38/2001, Universidade Federal Fluminense, Niterói.

(31) UFF (2001b). Relatório Final da Comissão Provisória de Alocação de Vagas Docentes. Universidade Federal Fluminense, Niterói.

(32) Valadares Tavares, L.; Themido, I.H.; Oliveira, R. \& Nunes Correia, F. (1996). Investigação Operacional. McGraw-Hill.

(33) Wei, Q.L.; Zhang, J. \& Zhang, X. (2000). An inverse DEA model for input/output estimate. European Journal of Operational Research, 121(1), 151-163.

(34) Yan, H.; Wei, Q. \& Hao, G. (2002). DEA models for resource reallocation and production input/output estimation. European Journal of Operational Research, 136, 19-31. 